\title{
光电协同效应降解饮用水中邻氯酚的机理 与动力学*
}

\author{
宋 强 曲久辉 ${ }^{* *}$ \\ (中国科学院生态环境研究中心环境水化学国家重点实验室, 北京 100085)
}

\begin{abstract}
摘要在集成光解电解一体化反应器中, 以邻氯酚(2-CP)为模型污染物, 通过产物和 动力学分析详细探讨了光电复合作用的协同效应机理, 建立了光电复合作用的动力学 模式. 表观动力学常数的对比表明复合协同作用对于总有机碳(TOC)的去除具有显著 的协同效应，研究表明，光电复合作用在研究条件下对污染物的降解具有多层次、多途 径的互补效应, 降解反应的途径不止是单独的光解与电解途径的简单累加, 而是通过 新的作用途径组合构成光电协同效应，通过紫外光辐射激发和定向直流电场作用控制 电极中毒和污染物分子的激发态及其迁移趋势，从而大大提高了降解反应的效率，通 过光电复合作用能够触发构成高级氧化的多种自由基链反应, 从而取得水中有机污染 物的快速、完全的矿化，动力学分析表明，光电协同作用在溶液中形成的羟基自由基 $(\mathrm{OH} \cdot)$ 反应是去除 TOC 的主要途径.
\end{abstract}

\section{关键词 邻攻䣲 光电协同效应 动力学 饮用水 微电解 $\mathrm{UV}$}

光催化技术和电催化技术作为高级氧化技术(AOPs)的重要内容, 在去除水中微量有机污 染物方面具有很好的应用前景. 光催化技术目前的不足主要是催化降解的速率尚不理想、光催 化剂的固化与稳定性等方面尚不成熟, 远未达到实用水平 ${ }^{[1 \sim 3]}$. 电催化技术用于有机化合物降 解的一个重要不足就是可能导致电极中毒的问题 ${ }^{[4]}$. 由于阳极直接电子得失生成的中间产物, 易于发生聚合或可逆反应, 并在电极表面形成不溶性聚合物膜使电极失去活性. 如何控制电 极中毒造成的失活一直是有机电解工业的难题之一 ${ }^{[4]}$. 控制电极污染的一般途径是选用复杂 的特种电极材料; 保持阳极电位在一个较低的正值, 也有利于控制电极被污染而抑制中毒 ${ }^{[5]}$. 最近的研究证明, 通过电场协助可有效提高光催化反应效率, 但技术经济性以及广谱适用性 方面存在缺陷 ${ }^{[6.7]}$. 其他的复合应用形式如光助电催化反应以及光解-电解复合的方法的研究 很少见于文献报道. 尽管有关单独的光解-光催化或电解-电催化过程降解水中有机污染物机 理已有大量研究, 但关于光电复合降解水中有机污染物的机理和动力学研究较少. 本文采用 低电压、微电流强度的直流(DC)电解和低压紫外(UV)光解联合的方法处理微污染饮用水, 提 出了外加短波长 UV 辐射的控制电极中毒的新途径, 并研究了光电复合降解水中邻氯酚(2-CP) 的协同增效效应的机理以及动力学规律, 为下一步改进电极材料和反应器设计从而提高降解

2002-10-15 收稿, 2002-12-27 收修改稿

* 国家自然科学基金杰出青年科学基金(批准号: 50225824)、中国科学院知识创新工程重要方向课题(批准号: KZCX2-409) 和国家“十五”科技攻关计划课题(批准号: 2001BA904B11)资助项目

** 联系人, E-mail: jhqu@mail.rcees.ac.cn 
速率提供理论依据.

\section{1 试验材料与方法}

\section{1 实验装置与药品}

实验装置即光电一体反应器如图 1 所示. 在同心圆筒反应器的中央放置紫外灯 $(253.7 \mathrm{~nm}$, $30 \mathrm{~W}), 0_{\mathrm{Cr}} 18_{\mathrm{Ni}} 9_{\mathrm{Ti}}$ 卫生级不锈钢制成的反应 器外筒体为阴极, $\mathrm{RuO}_{2} / \mathrm{TiO}_{2} / \mathrm{Ti}$ 网孔板卷制 而成的圆筒形的内筒体为阳极，极间距 $3 \mathrm{~cm}$, 反应器容积 $6.5 \mathrm{~L}$, 连接管路均为硅胶管. 待 处理水为蒸馏水配制的准确浓度 2-CP 的模 拟水溶液. 所用的试剂均为分析纯以上. 标 准溶液的配制和分析用水均为三次重蒸水.

\section{2 分析方法}

采用气相色谱 - 质谱联用 (5890IIGC5971MS, Hewlett Packard Co, 美国)分析 2-CP 及其降解产物. 同时检测总有机碳 (TOC)(Phoenix 8000 TOC Analyzer, TekmanDohrmann Co, 美国)和氯离子(离子色谱仪, Dionx 4500i, Dionx Co, 美国)指标.

\section{3 实验方案}

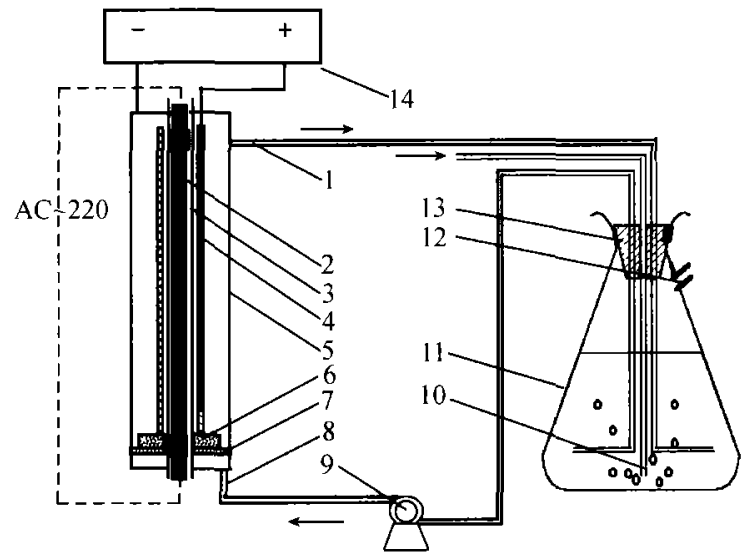

图 1 光电一体化反应器系统示意图 1. 活水管; 2. 紫外灯; 3. 石英衬管; 4. 阳极; 5. 阴极; 6. 聚㸨

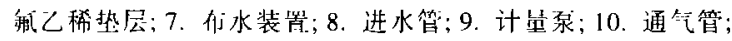
11. 淮被储䬱; 12. 取样11；13. 多孔寒; 14. 向流电源

运行方式为封闭循环, 定时在反应器出口处取样. 以 TOC 的去除速率和 2-CP 降解速率分 别为评价指标.

\section{2 结果与讨论}

\section{1 光电复合作用的表观反应速率常数与产物分析}

UV 光解、 DC 电解以及光电复合条件的降解反应, 分别以 2-CP 和 TOC 在不同反应时间 后的浓度比的负对数值对时间做图并线性拟合，得到的三种条件下的动力学方程如表 1 所示, 显然都符合拟一级动力学关系. 由表 1 可知, 以 2-CP 浓度为降解指标得到的表观反应速率常 数从大到小依次为: $0.0226 \mathrm{~min}^{-1}(\mathrm{UV}+\mathrm{DC})>0.0198 \mathrm{~min}^{-1}(\mathrm{UV})>0.0021 \mathrm{~min}^{-1}(\mathrm{DC})$, 可知 $\mathrm{UV}+$ DC 对 2-CP 的降解速率最大, 而 DC 对 2-CP 的降解速率最小; 而且 UV + DC 对 2-CP 的的表 观反应速率常数大于 UV 光解和 DC 电解两种单因素作用的和. 对 TOC 指标具有更加明显的 规律, UV + DC 复合作用条件对 TOC 的降解速率更远远超过 UV 光解和 DC 电解这两种单因 素的降解速率之和 $\left(0.014 \mathrm{~min}^{-1}>0.0072 \mathrm{~min}^{-1}+0.0023 \mathrm{~min}^{-1}=0.0095 \mathrm{~min}^{-1}\right)$ 达 $50 \%$. 这表明在 动力学上具有 UV 光解和 DC 电解的协同效应. UV 光解对邻氯酚的去除及脱氯反应作用明显, 而直流电解的脱氯反应速率较慢. DC 电解成为去除 TOC 的主要途径, 而光解对有机物的无机 化作用很小. 联用 UV + DC 能够大幅度提高 2-CP 的无机化速率, 表现出显著的协同效应. 与 文献中得到的其他光、电、声的高级氧化技术降解水中单氯酚类污染物的表观一级反应速率 常数的比较列于表 2, 可以看出光电复合的新技术具有很高的应用价值和发展前景. 
表 1 三种处理工艺对 2-CP 和 TOC 降解的表观动力学方程

\begin{tabular}{|c|c|c|}
\hline 处理条件 & 2-CP & TOC \\
\hline $\mathrm{UV}+\mathrm{DC}$ & $y=0.0226 x-0.0171, R^{2}=0.9914$ & $y=0.0014 x+0.1922, R^{2}=0.9159$ \\
\hline UV & $y=0.0198 x-0.1198, R^{2}=0.9856$ & $y=0.0023 x+0.0599, R^{2}=0.8336$ \\
\hline DC & $y=0.002 \mid x+0.0518, R^{2}=0.8434$ & $y=0.0072 x+0.0949, R^{2}=0.9524$ \\
\hline
\end{tabular}

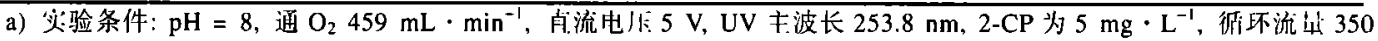
$\mathrm{mL} \cdot \mathrm{min}^{-1}$, 时问单位为 $\mathrm{min}$

表 2 多种高级氧化组合条件下单氯酚的表观降解反应速率常数

\begin{tabular}{|c|c|c|c|c|}
\hline 污染物 & 起始浓度 $/ \mathrm{mg} \cdot \mathrm{L}^{-1}$ & 降解条件 & 表观速率常数/ $/ \mathrm{min}^{-1}$ & 来源文献 \\
\hline \multirow[t]{5}{*}{$2-\mathrm{CP}$} & 11.06 & 超声i, pH 5.7 & 0.0048 & [8] \\
\hline & 5 & $\mathrm{UV} / \mathrm{TiO}_{2}$ & 0.00003391 & {$[9]^{\mathrm{a})}$} \\
\hline & 20 & $\mathrm{UV} / \mathrm{TiO}_{2}$ & 0.000448 & {$[10]$} \\
\hline & 400 & 电解, $\mathrm{Ti} / \mathrm{PbO}_{2}$ & 0.0075 & [11] \\
\hline & 600 & 电解, $\mathrm{Ti} / \mathrm{SnO}_{2}$ & 0.009 & [11] \\
\hline \multirow[t]{2}{*}{ 间氯酚 (3-CP) } & 10 & 超圭, $\mathrm{pH} 5.4$ & 0.0044 & [8] \\
\hline & 20 & $\mathrm{UV} / \mathrm{TiO}_{2}$ & 0.0004374 & [10] \\
\hline \multirow[t]{3}{*}{ 对氯酚(4-CP) } & 10 & 超声, pH 5.1 & 0.0033 & [8] \\
\hline & 20 & $\mathrm{UV} / \mathrm{TiO}_{2}$ & 0.0004586 & {$[10]$} \\
\hline & 153 & 电化学脱氯 & 0.0053 & [12] \\
\hline
\end{tabular}
的浓度 $(M)$

a) 根据 Tseng 等得H一个光催化降解 2-氯酚的反立速率常数的经验公式 $r=3.98 \times[\mathrm{C}]^{1.15}$ 计算得到, 式中 C 是 2-CP

降解 $30 \mathrm{~min}$ 后中间产物 GC-MS 分析谱图对比如图 2 所示. 由图 2 可见, 单独进行紫外光 解时, 有机物分子的化学键吸收能量而断开, 溶液中的中间产物较多, 主要是醌类或脱氯后被 氧络合的芳族化合物, 如苯醌、氢醌、邻苯二酚等, 2-CP(16.47 min) 降解较快. 单独进行直流 电解时，阳极降解反应可以通过阳极的直接电子转移和氧化水产生电生羟基自由基 $(\mathrm{OH} \cdot)$ 的途 径进行 ${ }^{[13]}$. 阳极得电子反应的产物可能发生聚合而导致电极中毒会抑制电解去除 2-CP 的速

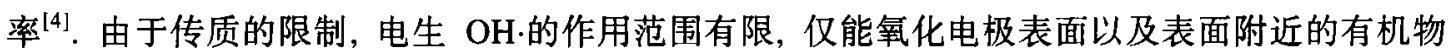
分子, 并直接将产生的有机中间产物氧化到 $\mathrm{CO}_{2}$, 反应较为彻底但速率有限 ${ }^{[11]}$. 由于是微电流 反应, 所以 2-CP 的去除较慢; 但可以测到的中间产物较少, 主要是马来酸、草酸等脂肪族羧 酸类物质. 光电复合作用下降解 2-CP 的产物在溶液中基本测不到. 因为光电复合作用能够直

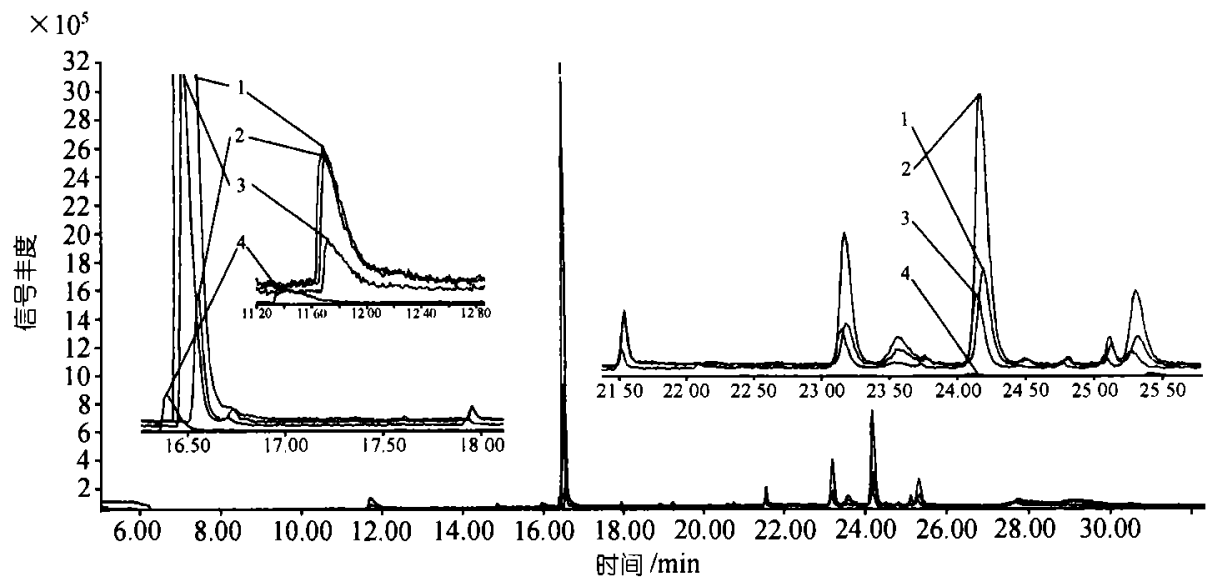

图 2 降解 $30 \mathrm{~min}$ 后的中间产物 GC-MS 谱图对比

1 示处理前水样; 2 示 UV 处理后水样; 3 示 DC 处理后水样; 4 示 UV + DC 处理厅水样 
接将有机污染物氧化为 $\mathrm{CO}_{2}$, 而不会象单独光解或电解或光催化过程产生大分子芳香族化合 物作为中间产物在溶液中的累积. 由于短波长的紫外辐射对溶液中的有机物分子有激发作用, 因此也会促进有机物分子更容易发生分解反应 ${ }^{[14]}$. 也可能在光电复合作用下的产物是少量水 溶性较强的小分子脂肪酸类物质, 在试样预处理中提取有损失的缘故 ${ }^{[4]}$.

\section{2 外加碳酸氢根对降解反应的影响}

为证实在光电复合作用下主要的降解途 径和强氧化性物种如羟基自由基有关，因此选 用典型的自由基捕获剂 ${ }^{[15]}$,也是天然水中常见 的成分重碳酸根加人到溶液中, 观察降解速率 是否受到影响. 在优选的反应条件下, 在待处 理溶液中添加碳酸氢钠达到 $3 \mathrm{mmol} / \mathrm{L}$ 时, 得 到的降解时间与三种指标的浓度关系如图 3 所 示. 由图 3 中可见, 加人碳酸氢根后 2-CP 的降 解速率略有减小, 而且氯离子的生成速率也与 2-CP 的消失速率相一致. 表明以脱氯为特征的 2-CP 的光解作用受到的影响并不显著. 但 TOC 的去除率则大大下降, 比不加碳酸氢根时 要小的多, 去除效果极不明显. 这表明, TOC 去除主要是通过自由基反应进行.

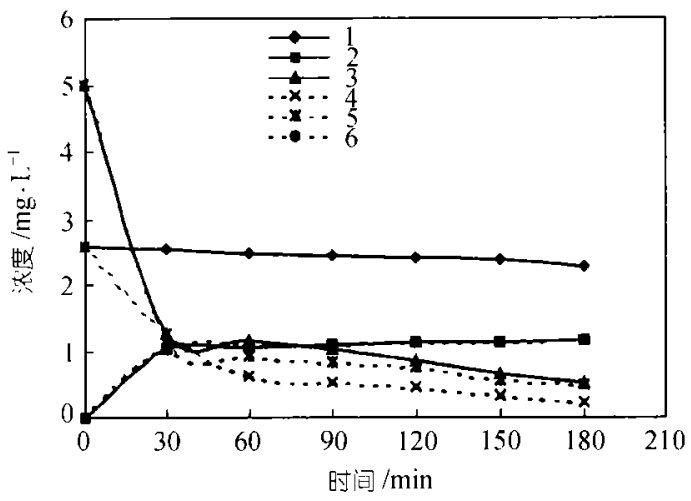

图 3 外加碳酸氢根时, 光电复合降解 2-CP 溶液 的降解时间与 [2-CP], [TOC], [Cl] 浓度的关系 $1 \sim 6$ 分别为: $T O C$ (加人重碳酸㭕); 氯离子(加人重湠酸概);

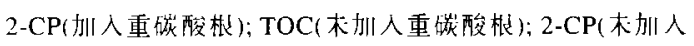

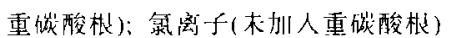

\section{3 电流效率}

假定理想的降解总反应为:

$$
\mathrm{ClC}_{6} \mathrm{H}_{4} \mathrm{OH}+27 \mathrm{OH} \rightarrow 6 \mathrm{CO}_{2}+16 \mathrm{H}_{2} \mathrm{O}+\mathrm{Cl}^{-}+26 \mathrm{e}^{-}
$$

假定发生反应的电荷量 $=$ 电流 $\times$ 时间, 则给定时间内的表观电流效率(apparent current efficiency, ACE) 为 ${ }^{[13]}$ :

$$
\mathrm{ACE}=\frac{\Delta(T O C)_{\text {实检值 }}}{\Delta(T O C)_{\text {埋论仕 }}} \times 100 \% .
$$

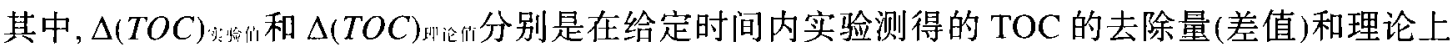
假定相同电量完全用于降解 TOC 的可去除量. 由(3)式可看出光电复合的电流效率明显大于单 独直流微电解时的电流效率:

$$
\mathrm{ACE}(\mathrm{UV}+\mathrm{DC})=72.21 \%>\mathrm{ACE}(\mathrm{DC})=49.6 \% .
$$

\section{4 光电复合作用-协同效应机理}

前面指出，光电复合条件下, TOC 去除主要是通过自由基反应进行，体相溶液中羟基自由 基 $(\mathrm{OH} \cdot)$ 的作用要比阳极氧化有效得多 ${ }^{\mid 131}$. 对比文献[1]可知，本文的 2-CP 的降解速率慢于 Fenton's Reagent 的氧化, 但快于 $\mathrm{UV} / \mathrm{TiO}_{2}$ 的速率, 降解曲线和 $\mathrm{UV} / \mathrm{H}_{2} \mathrm{O}_{2}$ 氧化的情况很接近, 而 且降解反应的中间产物也和典型的使用 $\mathrm{UV} / \mathrm{H}_{2} \mathrm{O}_{2}$ 的产物分布相似. 由于阳极不是光催化材料, 因此不能简单套用外加阳极偏压抑制光生电子-空穴复合的机理来解释; 即使电极材料中含有 $\mathrm{TiO}_{2}$ ，也没有证据表明阳极电氧化和光催化有关 ${ }^{[16]}$. 由于氧分子的还原电位高于氢离子的，因 此在阴极吸附的氧分子优先发生阴极还原反应生成 $\mathrm{H}_{2} \mathrm{O}_{2}$, 用 ${ }^{18} \mathrm{O}$ 同位素标记的实验已证实这 


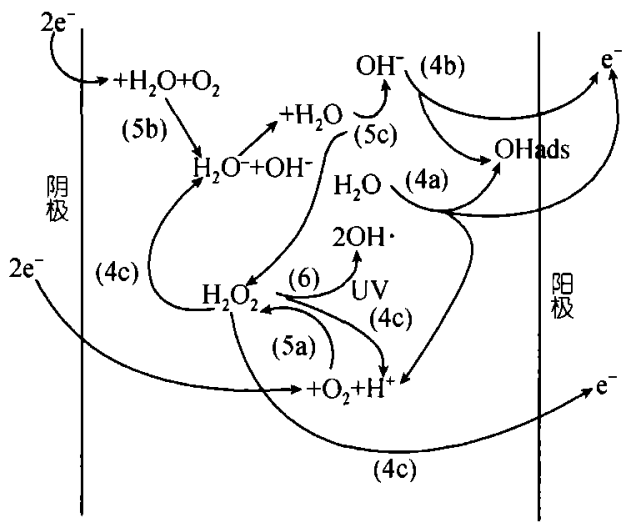

图 4 光电复合作用体系协同效应机理简图

一点 ${ }^{[17,18]}$. 因此, 本实验中外加 $U_{-254}$ 和 $5 \mathrm{~V}$ 电 压产生微电流, 在溶液中通人氧气, 保持一定浓 度的溶解氧(DO). DO 在阴极电极表面的吸附 态接受电子, 还原得到 $\mathrm{H}_{2} \mathrm{O}_{2}$. 为抑制氢离子的 竞争还原, 需要控制较低的阴极电极电位和保 持低的氢离子浓度 ${ }^{[18]}$. 由于光电复合的独特作 用, 必然存在多种产生自由基的途径, 包括电 生电极表面 $\mathrm{OH}^{\cdot[4.11,13]}$, 溶液体相中 UV 光激发 生成自由基等. 因此, 在复合的光电能量场下, 降解反应的途径不止是单独的光解与电解途径 的简单累加, 而是通过新的作用途径组合构成 光电协同效应, 如图 4 所示.

阳极反应: 阳极氧化水分子和氢氧根离子产生 $\mathrm{OH} \cdot$ 自由基 ${ }^{[11,13,19]}$ :

$$
\begin{aligned}
\mathrm{H}_{2} \mathrm{O} \rightarrow \mathrm{OH}_{\text {ads }}+\mathrm{H}^{+}+\mathrm{e}^{-} E_{0}=2.33 \mathrm{~V} & (4 \mathrm{a}) \\
\mathrm{OH} \rightarrow \mathrm{OH}^{\circ} \cdot \mathrm{ads}^{-} & (4 \mathrm{~b}) \\
& \mathrm{H}_{2} \mathrm{O}_{2} \rightarrow \mathrm{HO}_{2}+\mathrm{H}^{+}+\mathrm{e}^{-}
\end{aligned}
$$

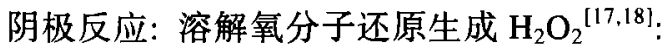

酸性环境:

$$
\mathrm{O}_{2}+2 \mathrm{H}^{+}+2 \mathrm{e}^{-} \rightarrow \mathrm{H}_{2} \mathrm{O}_{2}
$$

碱性环境:

$$
\begin{gathered}
\mathrm{O}_{2}+\mathrm{H}_{2} \mathrm{O}+2 \mathrm{e}^{-} \rightarrow \mathrm{OH}^{-}+\mathrm{HO}_{2}^{-} \\
\mathrm{HO}_{2}^{-}+\mathrm{H}_{2} \mathrm{O} \rightarrow \mathrm{H}_{2} \mathrm{O}_{2}+\mathrm{OH}^{-}
\end{gathered}
$$

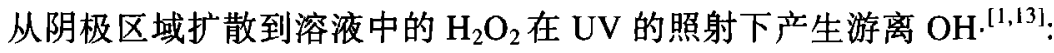

$$
\mathrm{H}_{2} \mathrm{O}_{2}+h v \rightarrow 2 \mathrm{OH} \text {. }
$$

光电复合作用下发生的各种降解反应式可表示如下:

$$
2-\mathrm{CP} \stackrel{k_{v v}}{\longrightarrow} \text { 产物 } 1+\mathrm{CO}_{2}+\mathrm{H}_{2} \mathrm{O} \text { (体相溶液中, 电场增效) }
$$

2-CP $\stackrel{k_{\mathrm{vx}}}{\longrightarrow}$ 产物 2 (阳极表面,电子转移, UV 辐射增效)

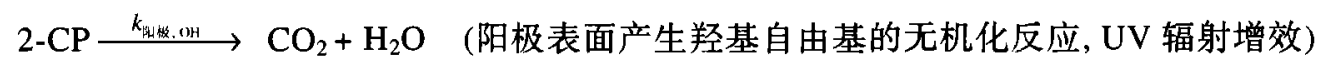

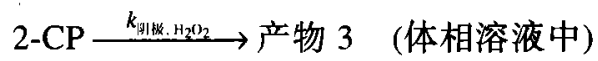

$$
\begin{aligned}
& \text { 2-CP } \stackrel{k_{\mathrm{UVH}} \mathrm{O}_{2}, \mathrm{HH} \cdot}{\longrightarrow} \mathrm{CO}_{2}+\mathrm{H}_{2} \mathrm{O} \text { (体相溶液中, UV-DC 复合增效) }
\end{aligned}
$$

反应(7)式表示电场增效的 UV 光解的反应. 有机物分子吸收了高于其离解能的紫外辐射, 发生断键. $\mathrm{UV}_{-254}$ 的灯管也会发出少量的更短波长的紫外辐射, 致使水分子裂解产生极微量的 $\mathrm{OH}^{[20]}$, 所以有微量的有机物被矿化. 反应(10)式表示在低电位下直流电解时阴极区生成的 $\mathrm{H}_{2} \mathrm{O}_{2}$, 直接氧化有机物分子. 由于定向直流电场的作用, 带电荷的污染物及中间产物粒子受稳 定的电场作用力作用, 从而能够抑制解离反应的复合以及光解的逆反应, 表现为电场的协同 增效.

反应(8)式表示直流电解的直接阳极有机物失电子反应. 反应(9)式表示直流电解阳极产生 $\mathrm{OH}$.氧化有机物分子. UV 照射在电极表面, 能够断开大分子链接 ${ }^{[21]}$, 防止电极中毒, 促进直接 阳极氧化水分子、氢氧根离子以及有机物分子, 从而提高了电流效率. UV 激发水溶液中的有 
机物分子到高能态, 更加易于发生反应. 反应(11)式表示短波长 UV 和阴极产生的 $\mathrm{H}_{2} \mathrm{O}_{2}$ 在体相 形成 $\mathrm{UV} / \mathrm{H}_{2} \mathrm{O}_{2}$ 产生 $\mathrm{OH}$.的强作用, 并激发自由基链反应, 是最重要的协同作用的途径之一. 溶 液中的 $\mathrm{OH}$.也包括极少量的由微量更短波长紫外线裂解水分子产生的和阳极表面吸附的 $\mathrm{OH}$.少量迁移到电极表面附近溶液中 ${ }^{[22]}$. 活性自由基如 $\mathrm{OH}$. 和有机污染物之间的活化能几近 于零, 因此氧化反应非常迅速, 氯代芳烃中的有机氯基团结构也能被有效破坏 ${ }^{[1]}$.

\section{5 光电复合协同作用的动力学模型}

根据前述机理分析, 在优化条件的光电复合作用下, 有机物降解的途径可由反应(7) (11) 式表示. 其中, 反应(10)式的作用相当小, 可以忽略不计 ${ }^{[23]}$. 其他途径中反应(8)和(9)式为表面 反应, 符合表面反应的动力学关系; 反应(7)和(11)式为溶液中的均相反应, 可用均相反应的动 力学关系表示. 因此, 光电复合降解作用的总速率可以表示为(假设光强度、2-CP 的吸光度、 水溶液的水力学条件等均保持恒定):

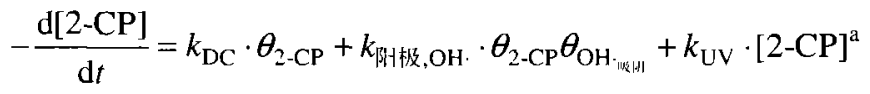

$$
\begin{aligned}
& +\left(k_{\mathrm{UV} / \mathrm{H}_{2} \mathrm{O}_{2}, \mathrm{OH}}+k_{\text {胡极近移 } \mathrm{OH} \cdot}+k_{\mathrm{UV}, \mathrm{OH}}\right) \cdot[2-\mathrm{CP}]^{\mathrm{b}} \cdot[\mathrm{OH} \cdot]_{\text {溶液中 }}^{\mathrm{c}}
\end{aligned}
$$

式中, 第一项代表电极表面由于有机物分子直接失去电子的氧化作用, $k_{\mathrm{DC}}$ 是该途径的反应速 率常数, 包含了电压、电极材料等的影响, $\theta_{2-\mathrm{CP}}$ 表示该反应物种吸附在电极表面的分数; 第二

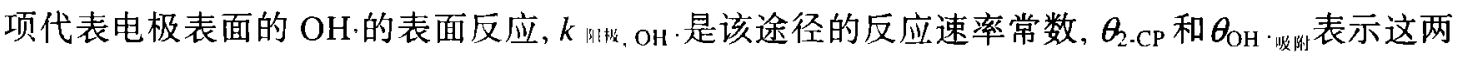
个反应物种吸附在电极表面的分数; 第三项代表 UV 光解作用, $k_{\mathrm{UV}}$ 是该途径的反应速率常数, 包含了光强、吸光度等的影响; 第四项代表光电复合条件下体相溶液中的 $\mathrm{OH}$. 的作用, $k_{\mathrm{UV} / \mathrm{H}_{2} \mathrm{O}_{2} . \mathrm{OH}}$. 是 $\mathrm{UV} / \mathrm{H}_{2} \mathrm{O}_{2}$ 途径的反应速率常数. $k_{\mathrm{UV}, \mathrm{OH}}$. 是极短波长 $\mathrm{UV}$ 裂解水途径的反应速率常 数. $k$ 阳打移, $\mathrm{OH}$. 是阳极表面吸附的 $\mathrm{OH}$-部分迁移到电极表面附近的溶液中途径的反应速率常数, 但该值极小 ${ }^{[4]}$. [2-CP], [OH· ]分别表示水溶液中物质的活度, 因是极低浓度溶液, 可以用浓度 值代替. $a, b, c$ 均为常数.

一般的高级氧化反应均为拟一级动力学, 本实验也表明动力学符合一级, 因此取 $\mathrm{a}$ 和 $\mathrm{b}$ 为 1. 反应器运行条件稳定后, 体相中产生的 $\mathrm{OH} \cdot$ 速率也恒定, 因此以常数 $M$ 表示:

$$
M=\left(k_{\mathrm{UV} / \mathrm{H}_{2}, \mathrm{O}_{2}, \mathrm{OH} \cdot}+k_{\text {陆极迁移 } \mathrm{OH}}+k_{\mathrm{UV}, \mathrm{OH}}\right) \cdot[\mathrm{OH} \cdot]_{\text {溶液中 }}^{\mathrm{c}}
$$

在电极材料、电压、溶液组成等条件为稳态时, 电极反应产生 $\mathrm{OH} \cdot$ 的速率以及阳极直接氧 化速率均保持不变, 在电极表面 2-CP(浓度极低, 可以忽略)和 $\mathrm{OH}$-的覆盖比率(由产生速率决 定)也保持稳定 ${ }^{[4,11,19]}$. 因此(12)式中的 1、2 项可以常数 $N$ 表示, 即

$$
N=k_{\mathrm{DC}} \cdot \theta_{2-\mathrm{CP}}+k_{\text {阴极, } \mathrm{OH}} \cdot \theta_{2-\mathrm{CP}} \cdot \theta_{\mathrm{OH}_{\text {M姆利 }}},
$$

记 $k_{\mathrm{UV}}$ 为 $K$, 则(12)式可以写成:

$$
-\frac{\mathrm{d}[2-\mathrm{CP}]}{\mathrm{d} t}=K \cdot[2-\mathrm{CP}]+M \cdot[2-\mathrm{CP}]+N,
$$

积分此式得到:

$$
[2-\mathrm{CP}]=\left(C_{0}+\frac{N}{K+M}\right) \cdot \mathrm{e}^{-t}-\frac{N}{K+M} .
$$

单独光解时, $N=0$, 所以有 


$$
[2-\mathrm{CP}]=C_{0} \cdot \mathrm{e}^{-t},
$$

为一级动力学方程, 与均相反应动力学关系相符.

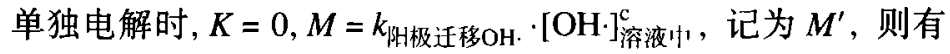

$$
[2-\mathrm{CP}]=\left(C_{0}+\frac{N}{M}\right) \cdot \mathrm{e}^{-t}-\frac{N}{M^{\prime}} .
$$

由于使有机物彻底无机化主要通过产生 $\mathrm{OH}$.的反应进行, 氧化剂可以归一化为用 $\mathrm{OH} \cdot$ 的 途径来表示. 还原剂则为有机污染物. 根据羟基自由基的不同来源途径和不同反应位置进行 推导. 因此有

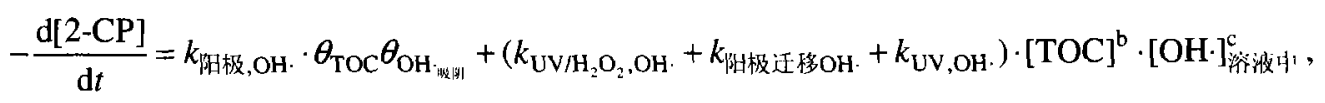

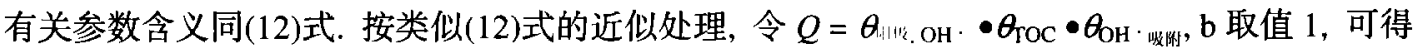

$$
-\frac{\mathrm{d}[\mathrm{TOC}]}{\mathrm{d} t}=M \cdot[\mathrm{TOC}]+Q
$$

积分可得

$$
[\mathrm{TOC}]=\left(C_{0}+\frac{Q}{M}\right) \cdot \mathrm{e}^{-t}-\frac{Q}{M} .
$$

单独光解时, $Q=0$, 所以有:

$$
[\mathrm{TOC}]=C_{0} \cdot \mathrm{e}^{-t},
$$

为一级动力学方程, 与均相反应动力学关系相符.

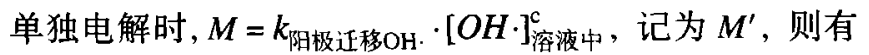

$$
[\mathrm{TOC}]=\left(C_{0}+\frac{Q}{M}\right) \cdot \mathrm{e}^{-t}-\frac{Q}{M^{\prime}} .
$$

以 2-CP 溶液初始浓度为 $5 \mathrm{mg} \cdot \mathrm{L}^{-1}$ ( TOC 为 $2.70 \mathrm{mg} \cdot \mathrm{L}^{-1}$ ), 时间单位为小时 $(\mathrm{h})$ 时的光电复 合条件下的降解实验数据进行处理, 将 2-CP 和 TOC 浓度分别对 $\exp (-t)$ 作图拟合, 分别得到两 条线性数据表达式, 为:

$$
\begin{array}{ll}
{[2-\mathrm{CP}]=5.4165 \exp (-t)-0.5334,} & R^{2}=0.9933 \\
{[\mathrm{TOC}]=2.7312 \exp (-t)-0.1164,} & R^{2}=0.9828
\end{array}
$$

显然(24)与(16)式、(25)与(21)式基本相符, 表明以上动力学推导符合实际情况. 根据(16)式, $\frac{N}{K+M}=0.5334$, 表明在光电复合作用去除 2-CP 中, 电极作用 $N$ 的贡献为 $K$ (光解) $+M$ (体相 $\mathrm{OH}$ ) 作用之和的一半, 显然电极作用对去除 2-CP 的贡献大大提高, 这是由于 UV 光照射电极 表面抑制电极中毒, 同时激发污染物分子更易于反应的光电协同作用的体现. 根据(21)式, $\frac{Q}{M}=0.1164$, 表明在 TOC 去除过程中, 电生 $\mathrm{OH}$ 的贡献 $Q$ 仅为 $M$ 作用的 $11.64 \%$, 主要的去除 作用是通过 $M$ 即体相溶液中 $\mathrm{OH} \cdot$ 的作用进行, 而电极过程产生差基自由基的途径作用很小. 这提示需要寻找更加有效的电极材料和电解参数, 以提高电极过程的催化氧化效率.此外, 提高 阴极 $\mathrm{H}_{2} \mathrm{O}_{2}$ 的产率, 提高协同效应的效率, 改进反应器设计.

\section{3 结论}

通过反应动力学模式的推导与验证以及产物分析, 可以得出如下结论: 
（1）光电复合条件下，存在显著的协同效应，尤其对 TOC 的去除效果明显大于光解、电解 单独作用之和。

(2) 光电协同效应主要体现在以下几点:

i) 短波长 UV 光照射电极能够有效控制电极表面污染，达到抑制电极中毒的效果;

ii) UV 激发有机物分子到更高的能态, 有利于发生各种裂解反应和通过 $\mathrm{OH}$ ·进行的高级氧 化反应;

iii）直流电场作用下，体系中带有相反电荷的污染物基团受到相反方向的电场力作用，从 而抑制光解反应的逆反应以及中间产物的聚合反应;

iv) 电解阴极区的溶氧还原为 $\mathrm{H}_{2} \mathrm{O}_{2}$, 扩散到体相溶液中, 与 UV 光照射构成 UV/ $\mathrm{H}_{2} \mathrm{O}_{2}$ 的 高级氧化组合, 产生的 $\mathrm{OH} \cdot$ 对溶液中 TOC 被迅速降解起主要作用.

(3) 动力学关系分析表明, 应该寻找更加有效的电催化材料, 提高电极表面反应对 TOC 降解的作用; 同时, 应该进一步改进反应器设计和优化运行参数.

\section{参考文献}

I Huang C P, Dong C, Tang Z. Advanced chemical oxidation: Its present role and potential future in hazardous waste treatment. Waste Management, 1993, 13:361 377

2 Johnson D C, Feng J, Houk L L. Direct electrochemical degradation of organic wastes in aqueous media. Electrochimica Acta, 2000, 46:323 330

3 Hoffmann M R, Martin S T, Choi W, et al. Environmental applications of semiconductor photocatalysis. Chem Rev, 1995. 95:69 96

4 Ezerskis Z, Jusys Z. Oxidation of chlorophenols on Pt electrode in alkaline solution studied by cyclic voltammetry, galvanostatic electrolysis, and gas chromatography-mass spectrometry. Pure Appl Chem, 2001, 73: 1929 1940

5 Rodgers J D, Bunce N J. Electrochemical treatment of 2,4,6-trinitrotoluene and related compounds. Environ Sci Technol, 2001, 35: 406 410

6 Butterfield I M, Christensen P A, Curtis T P, et al. Water disinfection using an immobilised titanium dioxide film in a photochemical reactor with electric film enhancement. Water Res, 1997. 31: 675 680

7 Hong L, Cheng S, Zhang J, et al. Titanium dioxide as photocatalyst on porous nickel: Adsorption and the photocatalytic degradation of sulfosalicylic acid. Chemosphere, 1999, 38: 283 287

8 Serpone N. Terzian R. Hidaka H, et al. Ultrasonic induced dehalogenation and oxidation of 2-, 3- and 4-chlorophenol in air-equilibrated aqueous media. Similarities with irradiated semiconductor particulates. J Phys Chem, 1994, 98: 2634 2640

9 Tseng J M, Huang C P. Removal of chlorophenols from water by photocatalytic oxidation. Wat Sci Tech, 1991, 23: 377 387

10 D'Oliveira J C. Al-Sayyed G, Pichat P. Photodegradation of 2- and 3-chlorophenol in $\mathrm{TiO}_{2}$ aqueous suspensions. Environ Sci Technol, 1990, 24: 990 996

II Polcaro A M. Palmas S, Renoldi F, et al. On the performance of $\mathrm{Ti} / \mathrm{SnO}_{2}$ and $\mathrm{Ti} / \mathrm{PbO}_{2}$ anodes on electrochemical degradation of 2-chlorophenol for wastewater treatment. J Appl Electrochem, 1999, 29: 147 151

12 Cheng I F, Fernando Q, Korte N. Electrochemical dechlorination of 4-chlorophenol to phenol. Environ Sci Technol, 1997, 31 : 1074 1078

13 Brillas E, Sauleda R, Casado J. Degradation of 4-chlorophenol by anodic oxidation, electro-fenton, photoelectro-fenton, and peroxy-coagulation processes. J Electrochem Soc, 1998, 145: 759 765

14 Yatmaz H C, Wallis C, Howarth C R. The spinning disc reactor-studies on a novel $\mathrm{TiO}_{2}$ photocatalytic reactor. Chemosphere, 2001, 42: 397 403

15 Cossu R, Polcaro A M, Lavagnolo $\mathrm{M} \mathrm{C}$, et al. Electrochemical treatment of landfill leachate: Oxidation at $\mathrm{Ti} / \mathrm{PbO}_{2}$ and 
$\mathrm{Ti} / \mathrm{SnO}_{2}$ anodes. Environ Sci Technol, 1998, 32: 3570 3573

16 Lidia S, Claudia J, Santosh N K. A comparative study on oxidation of disperse dyes by electrochemical process, ozone, hypochlorite and fenton regent. Wat Res, 2001, 35: 2129 2136

17 Brillas E, Calpe J C, Casado J. Mineralization of 2,4-D by advanced electrochemical oxidation processes. Wat Res, 2000, 34: 2253 2262

18 Chen J, Ollis D F, Rulkens W H, et al. Photocatalyzed oxidation of alcohols and organochlorides in the presence of native TiO2 and metalized $\mathrm{TiO}_{2}$ suspensions. Part ( II ): Photocatalytic mechanisms. Wat Res, 1998, 32: 669 676

19 Polcaro A M, Paimas S, Renoldi F, et al. Three-dimensional electrodes for the electrochemical combustion of organic pollutants. Electrochimica Acta, 2000, 46: 389 394

20 Shirayama H, Tohezo Y, Taguchi S. Photodegradation of chlorinated hydrocarbons in the presence and absence of dissolved oxygen in water. Wat Res, 2001, 35: 1941 1950

21 Rodgers J D, Bunce N J. Electrochemical treatment of 2,4,6-trinitrotoluene and related compounds. Environ Sci Technol, 2001, 35: 406 410

22 Panizza M, Bocca C. Cerisola G. Electrochemical treatment of wastewater containing polyaromatic organic pollutants. Wat Res, 2000, 34: 2601 2605

23 Shen Y S, Ku Y, Lee K C. The effect of light absorbance of the decomposition of chlorophenols by ultraviolet radiation and UV/H2O2 processes. Wat Res, 1994, 28: 907 914 